\title{
Divertículos duodenales periampulares y el fallo técnico-terapéutico durante colangiopancreatografía retrógrada endoscópica (CPRE)
}

\section{Periampullary duodenal diverticula and technical-therapeutic failure during endoscopic retrograde cholangiopancreatography (ERCP)}

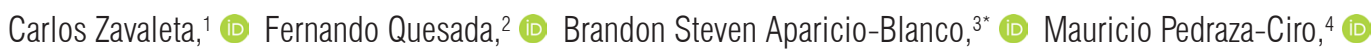

Andrés Mendoza-Zuchini, ${ }^{5}$ (b) Luis Felipe Cabrera. ${ }^{6}$ (D)

\author{
Gacceso abierto \\ Citación: \\ Zavaleta C, Quesada F, Aparicio-Blanco \\ BS, Pedraza-Ciro M, Mendoza-Zuchini \\ A, Cabrera LF. Divertículos duodenales \\ periampulares y el fallo técnico-terapéutico \\ durante colangiopancreatografía retrógrada \\ endoscópica (CPRE). Rev Colomb \\ Gastroenterol. 2021;36(4):539-543. https://doi. \\ org $/ 10.22516 / 25007440.706$ \\ Cirugia endoscópica, Intergastro. Bogotá, \\ Colombia. \\ Cirugia endoscópica, Intergastro. El Salvador. \\ Interno de medicina, Universidad El Bosque. \\ Bogotá, Colombia. \\ Residente de cirugía general, Universidad El \\ Bosque. Bogotá, Colombia. \\ 5 Cirugía general, Los Cobos Medical center. \\ Bogotá, Colombia. \\ 6 Cirugía general, fellow de cirugía vascular y \\ angiología, Universidad Militar Nueva Granada. \\ Bogotá, Colombia. \\ *Correspondencia: Brandon Aparicio \\ brandonaparici003@gmail.com \\ Fecha recibido: $18 / 12 / 20$ \\ Fecha aceptado: 22/02/21
}

\section{Resumen}

Introducción y objetivos: los divertículos duodenales periampulares (DDP) son infrecuentes y su hallazgo es incidental. Además, se clasifican en tres tipos según Boix por la cercanía a la ampolla mayor. El objetivo de este estudio fue realizar una descripción de nuestra experiencia con esta anomalía anatómica y demostrar la forma en que esto afecta la tasa de éxito técnica y terapéutica del procedimiento. Material y métodos: se realizó un estudio de corte transversal, con recolección de datos de forma retrospectiva en un período de 5 años de pacientes con divertículos periampulares duodenales. Se evaluó el fracaso terapéutico, complicaciones y dificultad de canulación de la vía biliar. Resultados: se evaluó un total de 214 pacientes, con una relación mujer-hombre de 2,15:1. La distribución de los sujetos por tipo de DDP fue: tipo 1 (29,9\%), tipo 2 (51,9\%) y tipo 3 (18,2\%). La indicación más frecuente de CPRE fue los cálculos del conducto biliar común en un 53,3\%. El DDP tipo 1 presentó mayor dificultad de canulación $(11,6 \%$ y falla terapéutica $(28,12 \%)$. Conclusión: la presencia de DDP durante la CPRE se asocia con una mayor falla técnica (falla en la canulación) y falla terapéutica (persistencia de la obstrucción biliar). Además, estas fallas aumentan considerablemente cuando se trata de una papila intradiverticular tipo 1 de la clasificación según Boix. Por lo anterior, se sugiere que los procedimientos endoscópicos biliares en estas condiciones sean realizados por endoscopistas con gran experiencia con el fin de minimizar la probabilidad de falla técnica y terapéutica, y las complicaciones asociadas.

\section{Palabras clave}

Divertículo duodenal, periampular, colangiopancreatografía retrógrada endoscópica, canulación, ampolla de Váter.

\section{Abstract}

Introduction and objectives: Periampullary duodenal diverticula are infrequent, and their finding is incidental. They are classified into three types according to Boix due to their proximity to the larger blister. This study aims to describe the experience with this anatomical abnormality and to demonstrate how this affects the technical and therapeutic success rate of the procedure. Materials y Methods: A cross-sectional study was conducted, with retrospective data collection over a 5-year period of patients with periampullary duodenal diverticula. Therapeutic failure, complications, and difficulty of cannulation of the bile duct were evaluated. Results: A total of 214 patients were evaluated, with a female-male ratio of 2.15: 1. The distribution of the subjects by type of PDD was: type $1(29.9 \%)$, type $2(51.9 \%)$, and type $3(18.2 \%)$. The most frequent indication for ERCP was common bile duct stones in 53.3\%. Type 1 PDD presented greater difficulty in cannulation $(11.6 \%)$ and therapeutic failure $(28.12 \%)$. Conclusion: The presence of PDD during ERCP is associated with greater technical failure (failure in cannulation) and therapeutic failure (persistence of biliary obstruction). In addition, this failures increases considerably when it is a type 1 intradiverticular papilla of the Boix classification. Therefore, it is suggested that biliary endoscopic procedures in these conditions are performed by highly experienced endoscopists to minimize the probability of technical and therapeutic failure and associated complications.

\section{Keywords}

Duodenal diverticula, Periampullar, Endoscopic retrograde cholangiopancreatography, Cannulation, papilla of Vater. 


\section{INTRODUCCIÓN Y OBJETIVOS}

Los divertículos gastrointestinales tienen su localización principalmente a nivel colónico y se ha observado que su segunda ubicación más frecuente es el duodeno, este último a nivel de la segunda y tercera porción duodenal ${ }^{(1)}$. Se denominan periampulares cuando están en un radio de 2 a $3 \mathrm{~cm}$ de la ampolla de Váter ${ }^{(2,3)}$.

Los divertículos duodenales periampulares (DDP) tienen una incidencia entre el $10 \%$ y el $15 \%$ en los pacientes a los que se les realiza una colangiopancreatografía retrógrada endoscópica (CPRE), su incidencia aumenta con la edad alcanzando hasta el $25 \%$ al $30 \%$ en pacientes entre los 50 y 60 años $^{(1,4,5)}$. En la mayoría de los casos, estos son asintomáticos ${ }^{(4)}$ y su diagnóstico es incidental ${ }^{(2)}$.

Los DDP se han clasificado en tres tipos según la relación entre la ampolla duodenal mayor y la variante anatómica ${ }^{(1)}$, además de que se asocia con un aumento en las fallas y complicaciones cuando se realiza canulación ${ }^{(4,6,7)}$; sin embargo, otros autores consideran que este procedimiento tiene los mismos riesgos con o sin este defecto ${ }^{(8)}$. Por lo anterior, decidimos revisar y describir nuestra experiencia con esta anomalía anatómica y demostrar el modo en que esto afecta la tasa de éxito del procedimiento.

\section{MATERIAL Y MÉTODOS}

\section{Población}

Se realizó un estudio observacional transversal, con recolección de datos de forma retrospectiva de registros médicos electrónicos, de pacientes con diagnóstico de DDP. La población de estudio fue los pacientes del centro Intergastro El Salvador, entre los años 2013 y 2017.

Se incluyeron pacientes mayores de 18 años con hallazgo incidental institucional de DDP y pacientes remitidos con diagnóstico de DDP para intervención endoscópica. Esta última fue realizada por 2 endoscopistas, quienes tienen un promedio de 750 a 900 CPRE anuales. Para el procedimiento, a todos los pacientes se les suministró lidocaína tópica bucofaríngea en aerosol al $10 \%$, sedación con midazolam (1-3 mg) o meperidina (30-50 mg) según el criterio del endoscopista y dosis única de antiespasmódico con butilbromuro de hioscina $(20 \mathrm{mg})$. El procedimiento se realizó con estricta monitorización del paciente, según los protocolos de la institución, el cual fue supervisado por el médico con certificación en sedación.

Los DDP se clasificaron según Boix (Tabla 1) $)^{(1)}$. La falla técnica se definió como problemas relacionados con la canulación y se clasificó según Boix ${ }^{(1)}$ y las pautas de la ESGE (Sociedad Europea de Endoscopia gastrointestinal) (Tabla 2). La falla terapéutica se consideró en los pacien- tes que durante el seguimiento persistieron con síndrome biliar obstructivo. Dentro de las complicaciones relacionadas con el procedimiento se tuvieron en cuenta el sangrado y pancreatitis pos-CPRE, la cual se definió como un dolor abdominal posterior al procedimiento con una amilasa en sangre mayor a 3 veces del límite de referencia ${ }^{(9)}$.

Tabla 1. Clasificación de los divertículos duodenales periampulares según Boix ${ }^{(1)}$

\begin{tabular}{|c|c|c|}
\hline Tipo & Descripción & Subtipos \\
\hline I & $\begin{array}{l}\text { Localización de la } \\
\text { papila dentro del } \\
\text { divertículo }\end{array}$ & $\begin{array}{l}\text { a. Hacia el costado superior } \\
\text { b. Hacia el costado izquierdo } \\
\text { c. Hacia el costado inferior } \\
\text { d. Hacia el costado derecho }\end{array}$ \\
\hline$\|$ & $\begin{array}{l}\text { Localización de la } \\
\text { papila hacia el borde } \\
\text { del divertículo }\end{array}$ & $\begin{array}{l}\text { a. Ubicado en el margen apical izquierdo } \\
\text { b. Ubicado en el margen apical derecho } \\
\text { c. Ubicado en el margen del centro } \\
\text { izquierdo o derecho } \\
\text { d. Papila ubicada entre el margen de dos } \\
\text { divertículos }\end{array}$ \\
\hline III & Diverti & o $3 \mathrm{~cm}$ de la papila \\
\hline
\end{tabular}

Tabla 2. Clasificación según la dificultad de canulación modificada según lo expuesto por Boix y las guías de la $\operatorname{ESGE}^{(1,18)}$

Grado Descripción

1 Canulación profunda fácil con métodos rutinarios

2 Requisito de alambre guía o esfinterótomo especial para lograr la canulación

3 Canulación difícil que requiere técnicas y habilidades especiales como la esfinterotomía con cuchillo de aguja Otras técnicas actuales basadas en las pautas de la ESGE: hemoclips, dilatación con balón del divertículo, colocación de stent pancreático, uso de videogastroscopio con tapa

4 Imposibilidad de canulación profunda

Este estudio fue aprobado por el comité de ética de nuestra institución. El protocolo fue realizado según las guías de ética médica World Medical Association Declaration of Helsinki-Ethical Principles for Medical Research Involving Human Subjects, adopted by the $18^{\text {th }}$ WMA General Assembly, Helsinki, Finland, June 1964, and revised in Tokyo 2004"(10).

\section{Recolección de información}

Se realizó la revisión retrospectiva de historias clínicas de los pacientes sometidos a CPRE con hallazgos de DDP, en las que se incluyeron variables como la edad, sexo, indicación preoperatoria dada por el centro de referencia, diag- 
nóstico endoscópico, variables quirúrgicas y complicaciones posquirúrgicas.

El análisis estadístico inferencial de comparación se realizó mediante la prueba de chi cuadrado $\left(\chi^{2}\right)$ considerando valores de $p<0,05$ como estadísticamente significativos para una diferencia.

\section{RESULTADOS}

En un período de 5 años, 214 pacientes tuvieron algún tipo de DDP con una incidencia de DDP del 4,7 \% en nuestra población. En la distribución por sexo, hubo mayor cantidad de mujeres $(68,2 \%)$, que de hombres $(31,8 \%)$, con una relación de 2,15:1; y una mediana de edad de 73 años (desviación estándar $[\mathrm{DE}]+16,08$ ).

La indicación más frecuente de CPRE fue coledocolitiasis en 114 pacientes $(53,3 \%)$ y la distribución de los sujetos por tipo de DDP fue: tipo 1: $29,9 \%$, tipo $2: 51,9 \%$ y tipo 3: 18,2 \% (Figura 1, Tabla 1). Dentro de las complicaciones se observó una relación entre la tasa de éxito técnico (canulación) y el tipo de DDP. Teniendo que el tipo 1 discriminada en grado de dificultad como grado $4(11,6 \%)$ y grado $3(21,74 \%)$, $p$ no significativa $(0,23)$ (Tabla 3$)$.

La tasa de falla terapéutica para cada tipo de DDP mostró una tasa más alta de falla para el tipo $1(28,12 \%)$, frente al tipo $2(9 \%)$ y el tipo $3(0 \%)$, y estadísticamente no es significativa $(p=0,35)$. El sangrado posterior al procedimiento se presentó en un total de 7 pacientes $(3,29 \%)$ y no hubo diferencia estadística de la tasa de complicaciones entre los tipos de DDP $(p=0,395)$ (Tabla 4). No se identificaron en este estudio otras complicaciones asociadas como pancreatitis pos-CPRE, perforación o colangitis.

\section{DISCUSIÓN}

La localización más frecuente de los DDP son a nivel periampular en alrededor del $75 \%{ }^{(4)}$. Según Boix, estos
Tabla 3. Tipos de dificultad de canulación

\begin{tabular}{|c|c|c|c|c|c|}
\hline \multirow[t]{2}{*}{ Boix tipo } & \multicolumn{4}{|c|}{ Dificultad } & \multirow[t]{2}{*}{ Total } \\
\hline & 1 & 2 & 3 & 4 & \\
\hline 1 & 25 & 16 & 15 & 8 & 64 \\
\hline 2 & 62 & 30 & 17 & 2 & 111 \\
\hline 3 & 31 & 4 & 4 & 0 & 39 \\
\hline Total & 118 & 50 & 36 & 10 & 214 \\
\hline
\end{tabular}

Tabla 4. Cantidad de complicaciones reportadas por CPRE

\begin{tabular}{|cccc} 
Boix tipo & \multicolumn{2}{c}{ Complicaciones } & Total \\
\cline { 2 - 3 } & Si & No & \\
\hline 1 & 2 & 62 & 64 \\
\hline 2 & 5 & 106 & 111 \\
\hline 3 & 0 & 39 & 39 \\
\hline Total & 7 & 207 & 214 \\
\hline
\end{tabular}

últimos se clasifican endoscópicamente en tres tipos de acuerdo con su ubicación en relación con la ampolla de Váter, de los cuales los tipos 1 y 2 a su vez se subclasifican en a, b, c y d (Tabla 1$)^{(1)}$. La tipificación más común según la literatura es la $3(55,8 \%)^{(6,11)}$, seguida del tipo $2(25,6 \%)$ y el tipo $1(18,6 \%)^{(6)}$; sin embargo, en nuestro estudio fueron más frecuentes el tipo $2(51,9 \%)$ y el $1(29,9 \%)$ que el $3(18,25 \%)$.

Los DDP son más predominantes en las mujeres que en los hombres ${ }^{(6,12,13)}$, como se demuestra en nuestro estudio con una relación de 2,15:1. La población que presenta esta alteración en su mayoría no refiere síntomas asociados y
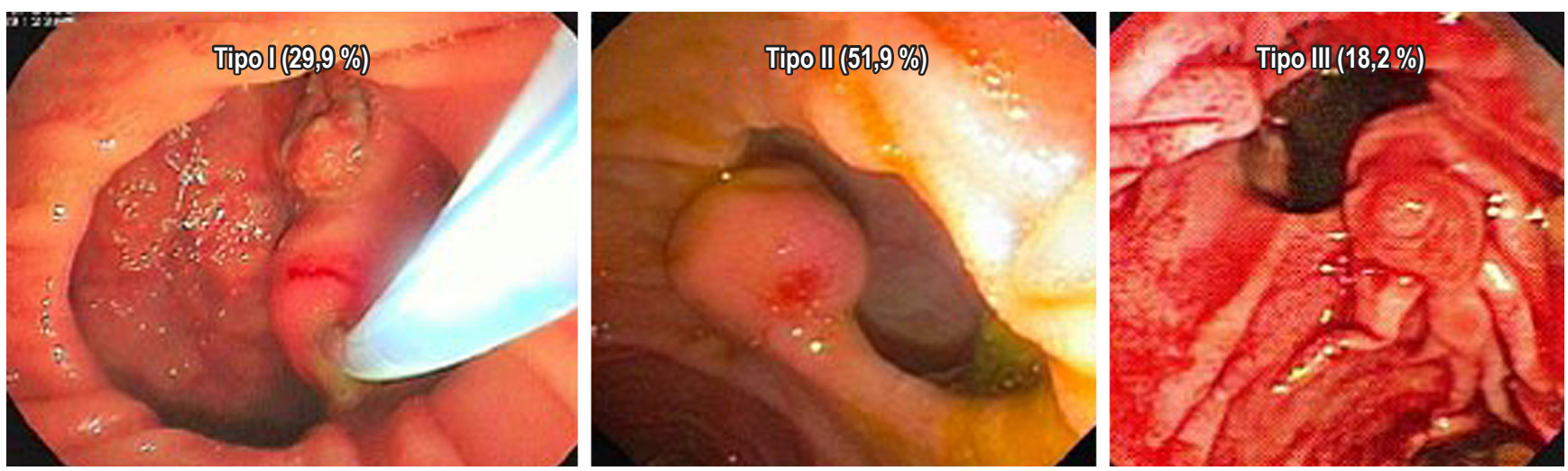

Figura 1. Distribución de los sujetos por tipo de DDP. 
el diagnóstico se consigue de manera incidental mediante imágenes radiográficas de forma menos frecuente o vía endoscópica durante la CPRE ${ }^{(6,7,14)}$. No obstante, pueden presentar sintomatología gastrointestinal inespecífica o específica en alrededor del $5 \%$, en la que se encuentra el dolor abdominal a nivel umbilical, epigástrico o en el hipocondrio derecho, cuadros eméticos y eructos, pero la presencia de estos no confirma el diagnóstico ${ }^{(15)}$.

Esta variante anatómica se asocia con cálculos del conducto biliar común, lo cual es atribuido y se fundamenta en varias teorías, dentro de las que se encuentran un mal funcionamiento del esfínter de Oddi, la compresión que puede generar el divertículo en el conducto biliar común en su porción distal o la presión aumentada en el tracto generada por espasmos papilares ${ }^{(6,16,17)}$. En el presente estudio se encontró una asociación entre DDP y litiasis biliar del $53,3 \%$ en concordancia con lo evidenciado en la literatura, pero también se ha relacionado con perforaciones, sangrado de la papila mayor, coledocolitiasis, pancreatitis $y$, en casos aislados, con tumores ${ }^{(6-8,11)}$.

La literatura expone que la canulación en pacientes con DDP tiene mayores complicaciones y fallas terapéuticas en comparación a los que se realiza la CPRE sin esta variación anatómica. Las dificultades que se encuentran son el aumento del tiempo, de los intentos, la experiencia y la habilidad del endoscopista ${ }^{(4,7)}$. Según las guías de la ESGE, se considera una canulación difícil cuando se tiene 5 contactos con la ampolla mayor y esta se intenta hacer por más de 5 minutos después de reconocer la estructura ${ }^{(18)}$. La tasa de fallo técnico es de alrededor del $61 \%$ al $95,4 \%{ }^{(6,7)}$ cuando existe esta variante anatómica, lo que indica una disminución en relación con los que no poseen esta alteración ${ }^{(6)}$. La principal causa de fallo técnico es la no identificación de la ampolla de Váter ${ }^{(6,7)}$ y esto se observa principalmente en los DDP tipo $1^{(6)}$, como se ve en nuestro estudio en un $8,4 \%$.

En un estudio retrospectivo realizado por Sfarti y colaboradores de 2016 a 2017 con un total de 98 pacientes, se dividieron en los grupos A (43 DDP) y B (55 no DDP), todos sometidos a CPRE. Con su grupo de estudio se demostró que las mujeres tienden a tener más riesgo con un Odds ratio (OR) de 1,099, al igual que lo documentado en nuestro estudio. Según la clasificación, el tipo 1 tuvo el $18,6 \%$, el tipo 2 tuvo $25,6 \%$ y el más frecuente fue el tipo 3 , con un $55,8 \%$. La canulación fácil fue menor en el grupo A $(62,8 \%)$ en comparación con el grupo B (83,6\%); adicionalmente, esta técnica fue más difícil en el grupo $\mathrm{A}$ $(25,6 \%)$ que en el grupo B $(16,4 \%)$. Las fallas terapéuticas fueron mayores en el grupo A (11,6\%), en relación con el grupo B $(0 \%)$. No existió diferencia significativa entre un grupo $\mathrm{u}$ otro en las complicaciones posteriores al procedimiento, similar a lo evidenciado en nuestro estudio ${ }^{(6)}$.

La principal limitación de nuestro estudio es que es un estudio observacional sin aleatorización, basado en una evaluación retrospectiva de una base de datos de nuestra institución.

\section{CONCLUSIÓN}

La presencia de DDP durante CPRE se asocia con mayor falla técnica (falla en la canulación) y falla terapéutica (persistencia de la obstrucción biliar). Además, estas fallas aumentan considerablemente cuando se trata de una papila intradiverticular tipo 1 de la clasificación según Boix, por lo cual sugerimos que los procedimientos endoscópicos biliares en estas condiciones sean realizados por endoscopistas con gran experiencia con el fin de minimizar la probabilidad de falla técnica, terapéutica y las complicaciones asociadas.

\section{REFERENCIAS}

1. Boix J, Lorenzo-Zúñiga $V$, Añaños F, Domènech E, Morillas RM, Gassull MA. Impact of periampullary duodenal diverticula at endoscopic retrograde cholangiopancreatography: a proposed classification of periampullary duodenal diverticula. Surg Laparosc Endosc Percutan Tech. 2006;16(4):208-11.

https://doi.org/10.1097/00129689-200608000-00002

2. Chen L, Xia L, Lu Y, Bie L, Gong B. Influence of periampullary diverticulum on the occurrence of pancreaticobiliary diseases and outcomes of endoscopic retrograde cholangiopancreatography. Eur J Gastroenterol Hepatol. 2017;29(1):105-111.

https://doi.org/10.1097/MEG.0000000000000744
3. Coman IS, Radu EV, Șandru V, Bârsan IC, Badiu CD, Constantinescu G, Grigorean VT; -. Retained Common Bile Duct Lithiasis at a Pacient with Periampullary Duodenal Diverticulum. Chirurgia (Bucur). 2016;111(6):517-521. https://doi.org/10.21614/chirurgia.111.6.517

4. Ospina Nieto J. Impacto verdadero del divertículo periampular en la CPRE. Rev Colomb Gastroenterol. 2007;22(4):297-301.

5. Sun Z, Bo W, Jiang P, Sun Q. Different Types of Periampullary Duodenal Diverticula Are Associated with Occurrence and Recurrence of Bile Duct Stones: A CaseControl Study from a Chinese Center. Gastroenterol Res 
Pract. 2016;2016:9381759.

https://doi.org/10.1155/2016/9381759

6. Sfarti VC, Bălan G Jr, Chiriac AŞ, Stanciu C, Bălan G, Gafencu-Şavlovschi D, Trifan AV. Endoscopic retrograde cholangiopancreatography (ERCP) in patients with periampullary diverticula. Rom J Morphol Embryol. 2018;59(3):833-837.

7. Karaahmet F, Kekilli M. The presence of periampullary diverticulum increased the complications of endoscopic retrograde cholangiopancreatography. Eur J Gastroenterol Hepatol. 2018;30(9):1009-1012. https://doi.org/10.1097/MEG.0000000000001172

8. Altonbary AY, Bahgat MH. Endoscopic retrograde cholangiopancreatography in periampullary diverticulum: The challenge of cannulation. World J Gastrointest Endosc. 2016;8(6):282-7. https://doi.org/10.4253/wjge.v8.i6.282

9. Gallego F, Gallardo F, Martínez G. Complicaciones de la colangiopancreatografía retrógrada endoscópica (CPRE). Identificación, prevención y manejo. PAPD. 2010; 33(1):22-36.

10. Declaración de Helsinki de la AMM-principios éticos para las investigaciones médicas en seres humanos [Internet]. AMM [consultado el 10 de junio de 2020]. Disponible en: https://www.wma.net/es/policies-post/declaracion-dehelsinki-de-la-amm-principios-eticos-para-las-investigaciones-medicas-en-seres-humanos/

11. Jakubczyk E, Pazurek M, Mokrowiecka A, Woźniak B, Małecka-Panas E, Podgórski M, Polguj M, Topol M. The position of a duodenal diverticulum in the area of the major duodenal papilla and its potential clinical implications. Folia Morphol (Warsz). 2021;80(1):106-113. https://doi.org/10.5603/FM.a2020.0012

12. Loffeld RJ, Dekkers PE. The Impact of Duodenal Diverticuli and the Execution of Endoscopic Retrograde Cholangiopancreaticography. Int Sch Res Notices.
2016;2016:5026289.

https://doi.org/10.1155/2016/5026289

13. Güitrón-Cantú A, Adalid-Martínez R, Gutiérrez-Bermúdez JA, Segura-López KF, García-Vázquez A. Grado de dificultad para canular papila de Vater en presencia de divertículo duodenal periampular. Rev Gastroenterol Méx. 2010;75(3):273-80.

14. Major P, Dembiński M, Winiarski M, Pędziwiatr M, Rubinkiewicz M, Stanek M, Dworak J, Pisarska M, Rembiasz K, Budzyński A. A Periampullary Duodenal Diverticula in Patient with Choledocholithiasis - Single Endoscopic Center Experience. Pol Przegl Chir. 2016;88(6):328-333. https://doi.org/10.1515/pjs-2016-0072

15. Mahajan S, Rajesh K, Upender C, Mokta J, Satinder M. Duodenal diverticulum: Review of literature. Indian Journal of Surgery. 2004;66(3):140-5.

16. Örmeci N, Deda X, Kalkan Ç, Tüzün AE, Karakaya F, Dökmeci A, Bahar DK, Özkan H, İdilman R, Çınar K. Impact of Periampullary Diverticula on Bile Duct Stones and Ampullary Carcinoma. Euroasian J Hepatogastroenterol. 2016;6(1):31-34. https://doi.org/10.5005/jp-journals-10018-1162

17. Lee JJ, Brahm G, Bruni SG, Thipphavong S, Sreeharsha B. Biliary dilatation in the presence of a periampullary duodenal diverticulum. Br J Radiol. 2015;88(1053):20150149. https://doi.org/10.1259/bjr.20150149

18. Testoni PA, Mariani A, Aabakken L, Arvanitakis M, Bories E, Costamagna G, Devière J, Dinis-Ribeiro M, Dumonceau JM, Giovannini M, Gyokeres T, Hafner M, Halttunen J, Hassan C, Lopes L, Papanikolaou IS, Tham TC, Tringali A, van Hooft J, Williams EJ. Papillary cannulation and sphincterotomy techniques at ERCP: European Society of Gastrointestinal Endoscopy (ESGE) Clinical Guideline. Endoscopy. 2016;48(7):657-83. https://doi.org/10.1055/s-0042-108641 